BMJ Open

Sport \&

Exercise

Medicine

\title{
CPR performance in the presence of audiovisual feedback or football shoulder pads
}

\author{
Shota Tanaka, ${ }^{1}$ Wayne Rodrigues, ${ }^{2}$ Susan Sotir, ${ }^{2}$ Ryo Sagisaka, ${ }^{3}$ \\ Hideharu Tanaka ${ }^{3}$
}

To cite: Tanaka S,

Rodrigues W, Sotir S, et al. CPR performance in the presence of audiovisual feedback or football shoulder pads. BMJ Open Sport Exerc Med 2017;3:e000208. doi:10.1136/bmjsem-2016000208

Accepted 9 April 2017

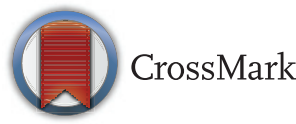

${ }^{1}$ Research Institute of Disaster management and EMS, Kokushikan University, Tama City, Tokyo, Japan ${ }^{2}$ Department of Exercise Science and Sports Studies, Springfield College, Springfield, Massachusetts, USA

${ }^{3}$ Department of EMS System, Graduate School, Kokushikan University, Tama City, Tokyo Japan

Correspondence to Mr Shota Tanaka, Research Institute of Disaster management and EMS, Kokushikan University; tanakamedical24@gmail.com

\section{ABSTRACT}

Objective The initiation of cardiopulmonary resuscitation (CPR) can be complicated by the use of protective equipment in contact sports, and the rate of success in resuscitating the patient depends on the time from incident to start of CPR. The aim of our study was to see if (1) previous training, (2) the presence of audiovisual feedback and (3) the presence of football shoulder pads (FSP) affected the quality of chest compressions.

Methods Six basic life support certified athletic training students (BLS-ATS), six basic life support certified emergency medical service personnel (BLSEMS) and six advanced cardiac life support certified emergency medical service personnel (ACLS-EMS) participated in a crossover manikin study. A quasiexperimental repeated measures design was used to measure the chest compression depth $(\mathrm{cm})$, rate $(\mathrm{cpm})$, depth accuracy (\%) and rate accuracy (\%) on four different conditions by using feedback and/or FSP Real CPR Help manufactured by ZOLL (Chelmsford, Massachusetts, USA) was used for the audiovisual feedback. Three participants from each group performed 2 min of chest compressions at baseline first, followed by compressions with FSP, with feedback and with both FSP and feedback (FSP +feedback). The other three participants from each group performed compressions at baseline first, followed by compressions with FSP+feedback, feedback and FSP.

Results CPR performance did not differ between the groups at baseline (median (IQR), BLS-ATS: 5.0 (4.4$6.1) \mathrm{cm}, 114(96-131) \mathrm{cpm}$; BLS-EMS: 5.4 (4.16.4) cm, 112(99-131) cpm; ACLS-EMS: 6.4 (5.76.7) $\mathrm{cm}, 138(113-140) \mathrm{cpm}$; depth $\mathrm{p}=0.10$, rate $p=0.37)$. A statistically significant difference in the percentage of depth accuracy was found with feedback (median (IQR), $13.8(0.9-49.2) \%$ vs 69.6 (32.3$85.8) \% ; p=0.0002)$. The rate accuracy was changed from $17.1(0-80.7) \%$ without feedback to 59.2 (17.3$74.3) \%$ with feedback $(\mathrm{p}=0.50)$. The use of feedback was effective for depth accuracy, especially in the BLSATS group, regardless of the presence of FSP (median (IQR), $22.0(7.3-36.2) \%$ vs $71.3(35.4-86.5) \%$; $\mathrm{p}=0.0002$ ).

Conclusions The use of audiovisual feedback positively affects the quality of the depth of CPR. Both feedback and FSP do not alter the rate measurements.

\section{What are the new finding?}

- The use of a feedback device was significantly effective, especially in the basic life support certified athletic training students group.

- Medically trained personnel are able to deliver the similar quality of CPR over the football shoulder pads for the first 2 min as suggested by the 2015 American Heart Association guidelines.

How might it impact on clinical practice in the near future?

- The use of a feedback device during chest compression-only CPR resulted in delivering a higher quality of CPR suggesting feedback devices must be introduced into athletic training settings.

- In case of a manpower shortage, athletic trainers should immediately initiate chest compressions over the football shoulder pads at least for the first 2 min until bystanders and automated external defibrillators are ready.

Medically trained personnel are able to deliver the desired depth regardless of the presence of FSP even though shallower chest compressions depth can be seen in CPR with FSP. A feedback device must be introduced into the athletic training settings.

\section{INTRODUCTION}

Out-of-hospital cardiac arrest (OHCA) is a global health concern and has grasped a lot of attention in the USA as 326200 people experience cardiac arrest per year. ${ }^{1}$ In Asian countries, OHCA has become an increasingly major cause of death. ${ }^{2}$ Public access to defibrillation through the use of automated external defibrillators (AEDs) has increased the survival likelihood. ${ }^{2-4}$ The 2015 American Heart Association (AHA) guidelines highlighted the first line of the OHCA Chain of Survival as recognition of the 
cardiopulmonary arrest (CPA) by bystanders. ${ }^{5}$ Although advanced life support (ALS) interventions are commonly believed to increase OHCA survival, high-quality bystander CPR is still a major component for improved survival rate. ${ }^{1}$ High-quality CPR is described as follows: rate between 100 and 120 compressions per min (cpm), compression depth between 5 and $6 \mathrm{~cm}$, allowing complete chest recoil after each compression, minimised interruptions in chest compressions and avoiding excessive ventilation.

Optimal chest compression depth has not yet been determined. ${ }^{6} 7$ Chest compressions with depth of $40 \mathrm{~mm}$ or less resulted in lower survival rates than depth of over $50 \mathrm{~mm} .{ }^{8}{ }^{9}$ The highest survival rate was found when the CPR providers gave $45.6 \mathrm{~mm}$ chest compressions. ${ }^{10}$ No significant difference was found in the range of compression depth between $40-53 \mathrm{~mm}$ manually and 50-60 mm mechanically through LUCAS2 manufactured by Physio Control (Redmond, Washington, USA). ${ }^{11}$ The compressions greater than $6 \mathrm{~cm}$ likely resulted in iatrogenic injuries. ${ }^{12}$ Increasing the chest compression depth with increased body mass index was suggested to maintain the persistent ratio of anteroposterior chest diameter to the compression depth. ${ }^{3}$ Even though the optimal chest compression depth has not been determined, performing highquality CPR is important to save a life. ${ }^{10}$ The use of feedback device is important to deliver high-quality CPR as improved CPR performance was seen with the use of the real-time visual and/or audible feedback. ${ }^{9}{ }^{14-}$ 17 The effectiveness of three different CPR devices was compared and only one device significantly increased the effectiveness of compressions. ${ }^{18}$ According to a study that compared the effectiveness of the depth and rate of CPR with and without laypersons using a feedback device for $3 \mathrm{~min}$ of chest compressions, the proportion of the depth was increased to $82 \%$ with feedback from $33 \%$ without feedback and the proportion of the rate was increased to $90 \%$ with feedback from $43 \%$ without feedback. ${ }^{19}$ Another study reported that feedback use significantly increased the compression quality during 2 min of chest compressions: $45.2 \%$ without feedback and $62.4 \%$ with feedback for depth, $73.1 \%$ without feedback and $94.6 \%$ with feedback for rate. ${ }^{20}$

At a sporting event, athletic trainers are trained as first responders and therefore responsible for initiating CPR. ${ }^{21}$ The lives of athletes depend on athletic trainers because the first $10 \mathrm{~min}$ following cardiac arrest is crucial. The survival chances decreased by $7 \%-10 \%$ every minute in a case where CPR and defibrillation were not administered. ${ }^{22}$ Cardiopulmonary resuscitation performance is complicated, however, in equipment intensive sports such as American football. Quality of CPR with football shoulder pads (FSP) on and off were studied. ${ }^{21}$

${ }^{23}$ Better CPR performance without FSP was reported. ${ }^{23}$ More adequate chest compression when the FSP were worn and the average time for removing the FSP to be $24.4 \mathrm{~s}$ were reported, but the researchers indicated lack of full chest recoil that would intern decrease blood flow. ${ }^{21}$ In cases of cardiac arrests during American football games, athletic trainers must expose the chest of the athlete in order to attach the AED pads by cutting the laces of the FSP. ${ }^{21}$ However, $70 \%$ of public secondary schools have medical coverage in the USA and $30 \%$ of them does not have any coverage during both games and practices, and multiple athletic trainers are employed at some schools. ${ }^{24}$ Removing FSP under the cardiac arrest situation by only one athletic trainer would be challenging.

The previous studies only focus on CPR performance of either emergency medical service (EMS) personnel or athletic trainers. The use of the feedback device improved the CPR performance in most of the studies. ${ }^{9}{ }^{14-17}$ Contrasting outcomes were seen in two studies of CPR performance with and without FSP. ${ }^{21} 23$ Current practice for initiating CPR when an athlete is wearing FSP is situation dependent. The effectiveness of training with or without FSP may affect CPR quality.

The purpose of our study was to see if (1) previous training, (2) the presence of audiovisual feedback and (3) the presence of FSP affected the compression depth and rate. The hypotheses of the study were that no difference in CPR quality between all groups would be found, that CPR quality would be significantly increase with the use of the feedback device and that all individuals would be able to perform the recommended depth of compressions accurately. The performing of highquality CPR by a single rescuer for a long period is not be easy because the quality of the chest compressions decreases as the rescuer's fatigues level increases. ${ }^{7}$ The use of audiovisual feedback devices therefore may increase CPR proficiency by helping to achieve compression depth for the required time.

\section{METHOD}

\section{Measurements}

Dependent variables included chest compression depth $(\mathrm{cm})$, rate $(\mathrm{cpm})$, depth accuracy $(\%)$ and rate accuracy (\%). Two AED Pro manufactured by ZOLL (Chelmsford, Massachusetts, USA) were used for the data collection of the chest compressions, and Real CPR Help is a software that gives real-time audiovisual feedback. One AED Pro was installed with Real CPR Help and used for a condition with feedback. The target zone for feedback was set according to the 2015 AHA guidelines. For the condition without feedback, the other AED Pro was used without Real CPR Help. A sensor was also included; CPR Statpadz uses accelerometer technology with sensors inside the CPR electrodes that measure the depth and rate of every compression. The captured data were analysed with RescueNet Code Review software. Depth was calculated as the depth of each compression divided by the number of compressions per second. Rate was calculated as the rate of each compression divided by the number of compressions per second. The number of performed compressions within the target zone of depth of between 


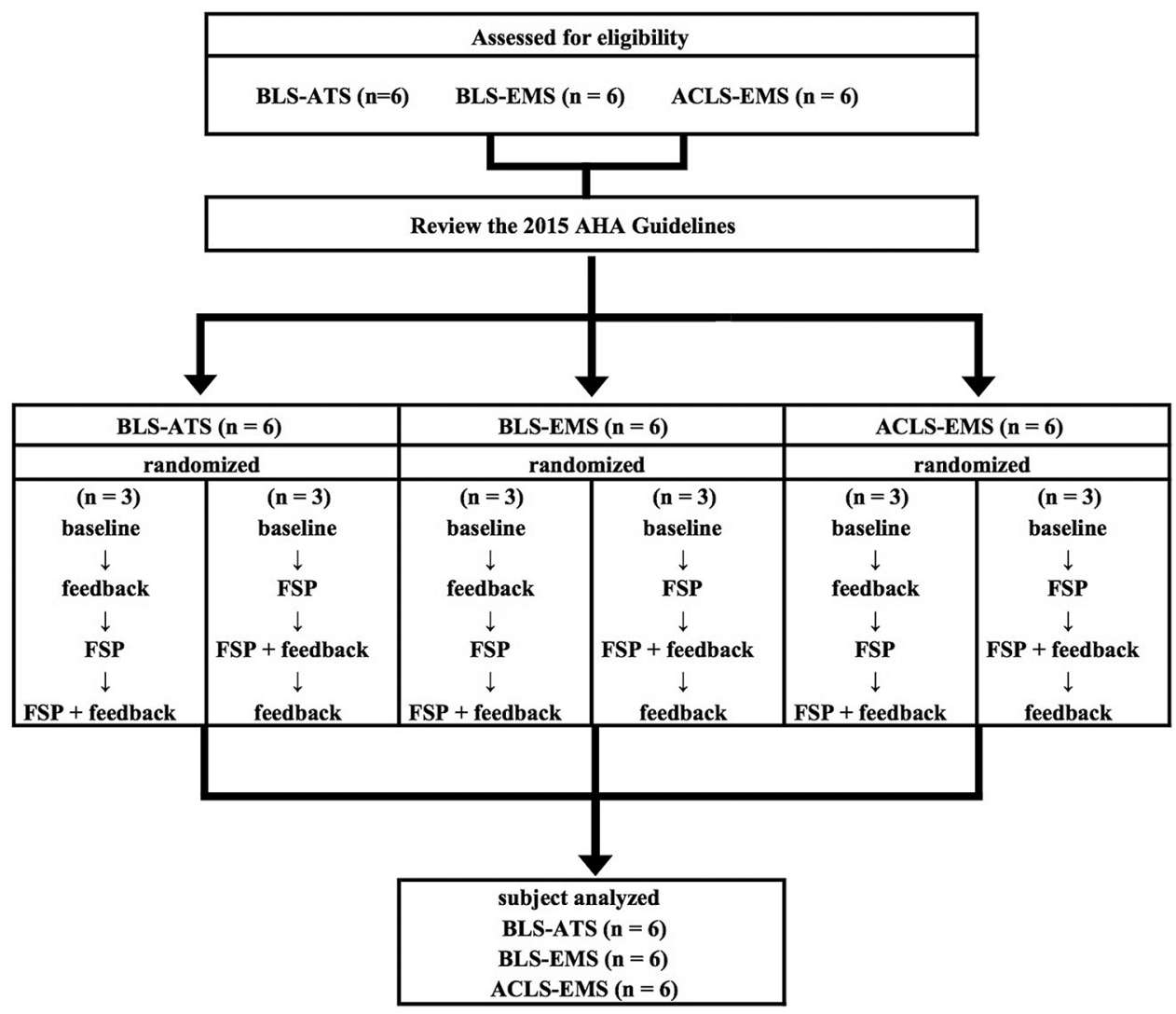

Figure 1 Flow chart of the study procedure.

5 and $6 \mathrm{~cm}$ divided by the total number of compressions was equal to the depth accuracy. Rate accuracy was calculated as the number of performed compressions within the target zone of a rate between 100 and $120 \mathrm{~cm}$ divided by the total number of compressions. A quasi-experimental repeated measures design was used for this study. Participants performed chest compressions on four different conditions, including the baseline measurements in the lab setting. In our study, a 1 min break was taken between each condition and the participant's fatigue level was not measured.

\section{Participants}

The Institutional Review Board at Springfield College approved the initiation of the study as of 1 February 2016. A total of 18 people participated in the study and were divided into three groups as follows:

1. BLS-ATS $(n=6)$ : Six basic life support (BLS) certified athletic training students (ATS) who were in the athletic training program accredited by the Commission on Accreditation of Athletic Training Education at Springfield College.

2. BLS-EMS $(n=6)$ : Six BLS certified EMS personnel.

3. ACLS-EMS $(n=6)$ : Six different EMS personnel who certified advanced cardiovascular life support (ACLS).

The inclusion criteria for all groups were a valid BLS-healthcare provider cardholder and no upper extremity injury in the past 6 months. For the BLSEMS group, EMS personnel who had become emergency medical technicians within the past 3 years were recruited. For the ACLS-EMS group, EMS personnel who had received an ACLS provider credential within the past 3 years were recruited. Any ATS who had any EMS background or ACLS certification were excluded. A power analysis was not completed to assess the number of participants needed per group. The independent variables were the three certification groups: BLS-ATS, BLS-EMS and ACLS-EMS.

\section{Instrumentation}

A Little Anne CPR manikin manufactured by Laerdal (Stavanger, Norway) was used in the study. The FSP were fitted according to the manufacturer's guidelines (figure 1). Data collection on chest compressions was measured with the use of AED Pro. The audiovisual feedback system, Real CPR Help, was installed. The CPR Stat-padz was placed as the 2015 AHA guidelines recommended. A compressing depth of $5-6 \mathrm{~cm}$ and rate at $100-120 \mathrm{cpm}$ are defined in the 2015 AHA guidelines, whereas a compressing depth over $5 \mathrm{~cm}$ and rate of over $100 \mathrm{cpm}$ are defined in the 2010 AHA guidelines. Real CPR Help gave feedback both visually and audibly. Due to the timing of the start date of the data collection, audio feedback did not 

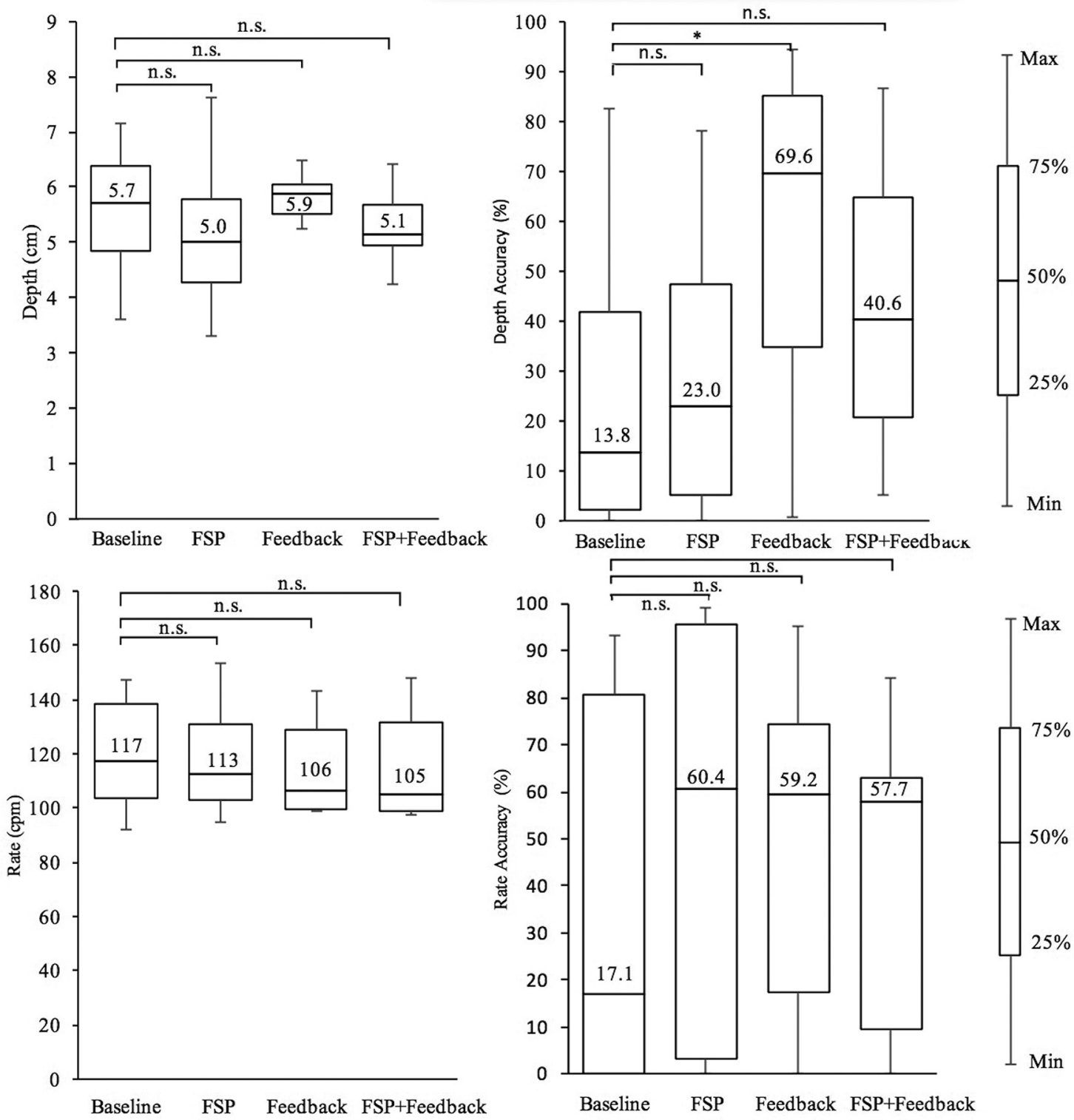

Figure 2 The depth and rate measurements of total of 18 participants on four different conditions. Baseline measurement was as the control measurement and multiple comparison was conducted. $p$ Value was corrected according to the Dunnett method. ${ }^{*} p=0.002$, n.s., not significant.

followed the 2015 AHA guidelines. For the depth measurement, Real CPR Help gave $5-6 \mathrm{~cm}$ of visual feedback as suggested by the 2015 AHA guidelines, but audio feedback followed the 2010 AHA guidelines. For the rate, the metronome beep was only set at $100 \mathrm{cpm}$. Real CPR Help gives audio feedback, such as 'push harder', 'good compressions' and 'continue CPR.'

\section{Procedures}

A quasi-experimental repeated measures design was used to examine how the quality of CPR performance by trained medical personnel changes when chest compressions were performed with the following conditions: audiovisual feedback and FSP. Participants were eligible to take part in the study after completing informed consent and a demographic form. After completing the demographic form, the participants visually and verbally reviewed the recommendations of current 2015 AHA guidelines.

A total of four conditions were performed. Chest compression-only CPR was performed for $2 \mathrm{~min}$ for each condition in the study, with $1 \mathrm{~min}$ of rest. Participants were asked to perform chest compressions under three different conditions after the baseline measurements. To complete baseline measurements, the participants started $2 \mathrm{~min}$ of chest compressions without audiovisual feedback and FSP. The three conditions are as follows:

1. Two minutes of chest compression-only CPR with audiovisual feedback

2. Two minutes of chest compression-only CPR with FSP 
Table 1 General background characteristics and baseline CPR performance

\begin{tabular}{llllll}
\hline & Total & BLS-ATS & BLS-EMS & ACLS-EMS & p Value \\
\hline Male, \% (Nb/total Nb) & $55.6(10 / 18)$ & $50.0(3 / 6)$ & $33.3(2 / 6)$ & $83.3(5 / 6)$ & N/A \\
CPR experience, \% (Nb/total Nb) & $33.3(6 / 18)$ & $16.7(1 / 6)$ & $16.7(1 / 6)$ & $66.7(4 / 6)$ & N/A \\
Depth, median (IQR), cm & $5.7(4.7-6.4)$ & $5.0(4.4-6.1)$ & $5.4(4.1-6.4)$ & $6.4(5.7-6.7)$ & 0.10 \\
Rate, median (IQR), cpm & $117(103-139)$ & $114(96-131)$ & $112(99-131)$ & $138(113-140)$ & 0.37 \\
\hline
\end{tabular}

Kruskal-Wallis test.

ACLS, advanced cardiovascular life support; ATS, athletic training students; BLS, basic life support; cpm, compression per min; EMS, emergency medical service.

3. Two minutes of chest compression-only CPR with both FSP and feedback (FSP+feedback)

All participants began the study with the baseline condition and achieved through the specific order of implementation. Three participants from each group performed chest compression at baseline first, followed by compression with feedback, with FSP and with FSP + feedback. The other three participants from each group performed at baseline first, followed by compression with FSP, with FSP+feedback and with feedback.

\section{Statistical analysis}

For the baseline, descriptive statistics were calculated for all demographic and dependent variables. The Kruskal-Wallis test was used for continuous variables while multiple-way analysis of variance was used for the analysis of depth and rate accuracy. The Wilcoxon test was used to evaluate how the independent variables affect the dependent variables. In figure 2, multiple comparisons were conducted and the Dunnett Method was used to correct $\mathrm{p}$ values. The level of significance for decision making was set at $\alpha=0.05$. Data were analysed by JMP V. 11.2.0 (the SAS Institute).

\section{RESULTS}

\section{Baseline characteristics of participants}

Eighteen participants, 10 male and eight female, were recruited (male: $55.6 \%$; table 1). One participant from the BLS-ATS group, one participant from the BLSEMS group and four participants from the ACLS-EMS group had experience with CPR in the field. The ACLS-EMS group had a higher percentage of CPR experience (CPR experience: 66.7\%; table 1). The background characteristics of the participants in the three groups and their baseline CPR performance are shown in table 1 .

\section{Depth measurements}

The median depth measures of all participants were as follows: $5.7 \mathrm{~cm}$ at baseline, $5.0 \mathrm{~cm}$ on FSP, $5.9 \mathrm{~cm}$ on feedback and $5.1 \mathrm{~cm}$ on $\mathrm{FSP}+$ feedback, respectively (figure 2). The median depth accuracies were as follows: $13.8 \%$ on baseline, $23.0 \%$ on FSP, $69.6 \%$ on feedback and $40.6 \%$ on FSP+feedback, respectively (figure 2). There were no interactions in the performance quality of depth accuracy between the groups and the use of the feedback device, between groups and the use of FSP and between the use of the feedback device and the use of FSP (table 2).

\section{Groups}

Both the BLS-ATS and BLS-EMS groups compressed to the depth as suggested by the 2015 AHA guidelines, and the median values were similar (table 1 ). As the effectiveness of feedback was shown by groups, the BLS-ATS group significantly increased depth accuracy to 71.3 (35.4-86.5)\% from $22.0(7.3-36.2) \%(\mathrm{p}=0.002$; table 3$)$. The ability to deliver accuracy in the depth of chest compressions between the three groups was not statistically different regardless of interventions (table 2).

\section{FSP and feedback}

FSP do not affect depth measurements, but the feedback device affects depth accuracy (table 2). With the use of FSP, the compression depth was significantly shallower than baseline, as all participants performed $5.7(4.7-6.4) \mathrm{cm}$ at baseline and $5.0(4.2-5.8) \mathrm{cm}$ with FSP $(\mathrm{p}=0.104$; figure 2$)$. The depth accuracies were as follows: $13.8(0.9-49.2) \%$ at baseline and 23.0 (2.3$50.7) \%$ with FSP ( $p=0.991$; figure 2$)$. The FSP did not significantly alter the quality of the depth of the chest compression measurements. As shown in figure 2 , the depth accuracies were as follows: $13.8(0.9-49.2) \%$ at baseline and $69.6 \quad(32.3-85.8) \%$ with feedback $(p=0.002)$. The use of feedback significantly affects the quality of the chest compression depth.

\section{Rate measurements}

The median rate measures of all participants were as follows: $117 \mathrm{cpm}$ at baseline, $113 \mathrm{cpm}$ with FSP, 106 cpm with feedback and $105 \mathrm{cpm}$ with FSP+feedback (figure 2). The median rate accuracies were as follows: $17.1 \%$ at baseline, $60.4 \%$ with FSP, $59.2 \%$ with feedback and $57.7 \%$ with FSP+feedback, respectively (figure 2). There were no interactions in the performance quality of rate accuracy between the groups and the use of the feedback device, between the groups and 
Table 2 Accurate percentage of depth and rate among the interventions

\begin{tabular}{lll}
\hline & \multicolumn{2}{l}{ ANOVA p value } \\
\cline { 2 - 3 } & $\begin{array}{l}\text { Depth } \\
\text { accuracy }\end{array}$ & $\begin{array}{l}\text { Rate } \\
\text { accuracy }\end{array}$ \\
\hline Group & 0.48 & 0.06 \\
\hline Feedback & $0.0003^{\star}$ & 0.68 \\
\hline Shoulder pad & 0.41 & 0.59 \\
Group $\times$ feedback & 0.40 & 0.99 \\
Group $\times$ shoulder pad & 0.86 & 0.99 \\
\hline Feedback $\times$ shoulder pad & 0.25 & 0.21 \\
\hline
\end{tabular}

Multiway ANOVA.

* $p<0.05$ significant.

ANOVA, analysis of variance.

the use of FSP and between the use of the feedback device and the use of FSP (table 2).

\section{Groups}

No statistically significant difference was found between the groups (table 2). The ACLS-EMS group compressed at $138 \mathrm{cpm}$ at baseline, which was faster than the 2015 AHA guidelines recommend (table 1). The BLS-ATS and BLS-EMS groups compressed within the desired rate regardless of audiovisual feedback.

\section{FSP and feedback}

The compression rate measurement was not affected by both FSP and feedback (table 2). Participants recorded $17.1(0-80.7) \%$ at baseline and $60.4(3.1-95.4) \%$ on FSP $(\mathrm{p}=0.44$; figure 2). Participants performed 59.2 (17.3$74.3) \%$ with feedback $(p=0.50$; figure 2$)$.

\section{DISCUSSIONS}

In the study, chest compression depth, rate and accuracies were measured in 18 different participants from the BLS-ATS, BLS-EMS and ACLS-EMS groups. CPR quality and compression depth were not different between all groups (table 2). No significant difference in CPR performance was found between first responders and EMS personnel groups. Medically trained personnel were able to perform an appropriate chest compression rate and depth accuracies regardless of the conditions (table 2). With the use of feedback, all participants performed high-quality chest compression depth regardless of the presence of FSP. Feedback affected to chest compression depth accuracy among the groups $(p=0.0003$; table 2). Through the findings, we found that the use of feedback device was proficient for performing the recommended depth (figure 2). As shown in figure 2, we found the use of the feedback device significantly increased depth accuracy by $55.8 \%$ as all participants performed at $13.8(0.9-49.2) \%$ under the baseline condition and $69.6(32.3-85.8) \%$ under the feedback condition ( $p=0.002$; figure 2$)$. In our study, the rate accuracy was increased by $42.1 \%$ from $17.1(0$ $80.7) \%$ to $59.2(17.3-74.3) \%(\mathrm{p}=0.50)$, where the former study reported that the average quality of compressions increased from $24 \%$ to $53 \%{ }^{14}$ In previous studies, researchers have stated the importance of using a feedback device, and recommended its use based on the supported effectiveness of the feedback device. ${ }^{9}{ }^{14-17}$

Throughout 2 min of chest compression-only CPR, a higher percentage of depth and rate accuracy was seen as participants were instructed to follow the 2015 AHA guidelines. No statistical differences between groups were found in any measurement. A recent study investigated $8 \mathrm{~min}$ of chest compression-only CPR. Maintaining high-quality CPR is the best that rescuers can do because rescuers experience increasing fatigue, resulting in the increased difficulty in meeting the 2010 AHA guidelines. $^{7}$ In one study, the mean compression depth declined over $8 \mathrm{~min}$, while the mean compression rate was consistently maintained. ${ }^{7}$ Another recent study compared the effectiveness of compressions between continuous chest compressions and standard CPR, and reported that the force of compressions was affected by fatigue; adequate compressions were delivered through continuous chest compressions rather than standard CPR during a 9 min period. ${ }^{25}$ A previous study found that a standard CPR group performed a higher rate of adequate chest compressions after $2 \mathrm{~min}$ and a higher number of adequate chest compressions over $8 \mathrm{~min}$ when compared with compression-only CPR. ${ }^{26}$ However, regardless of the continuous chest compression rate, subjects performed 103 to $108 \mathrm{cpm}$ over $9 \mathrm{~min}^{25}$ In our study, a $1 \mathrm{~min}$ break was taken between each condition and a fatigue level was not manifested.

Similar to our study, performing CPR on FSP resulted in a shallower chest compression depth (figure 2) ${ }^{23}$ Due to the fact that the chest compression with FSP was greater than $5.0 \mathrm{~cm}$ of depth, initiating chest compression over FSP could be effective in case of the complication of chest exposure. In order to remove FSP, $24.4 \mathrm{~s}$ was taken. ${ }^{21}$ The ideal on-field procedure is to expose the chest and apply AED as soon as possible, but medically trained personnel are still able to deliver the similar quality of CPR for the first 2 min as suggested by the 2015 AHA guidelines. As shown by comparing the groups in table 3 , the use of a feedback device was significantly effective, especially in the BLS-ATS group. More frequent CPR training is recommended and real-time feedback devices must be placed with AEDs in the field of athletic training. The BLS-ATS groups are first responders, but they will face cardiac arrest situations only rarely. Athletic trainers, however, face a higher possibility of responding to cardiac arrest though still not very often this the use of a feedback device is critical for performing high-quality CPR and increasing the survival rate. We also suggest practising CPR on the FSP for 
Table 3 The comparison in the performance difference on two interventions by groups

\begin{tabular}{|c|c|c|c|c|c|c|}
\hline & FSP OFF & FSP ON & p Value & Feedback OFF & Feedback ON & p Value \\
\hline \multicolumn{7}{|c|}{ Depth accuracy (\%) } \\
\hline BLS-ATS & $35.2(16.8-85.3)$ & $37.6(21.9-57.8)$ & 0.56 & $22.0(7.3-36.2)$ & 71.3 (35.4-86.5) & $0.002^{*}$ \\
\hline BLS-EMS & 40.6 (1.9-71.2) & $26.1(6.9-56.7)$ & 0.64 & $9.3(0.4-53.2)$ & 45.1 (18.6-69.7) & 0.06 \\
\hline ACLS-EMS & 38.2 (3.2-79.2) & 37.5 (18.9-69.7) & 0.82 & $22.0(4.4-59.9)$ & $58.8(17.6-78.8)$ & 0.18 \\
\hline \multicolumn{7}{|l|}{ Depth (cm) } \\
\hline BLS-ATS & $5.4(4.9-6.1)$ & $5.1(4.5-5.2)$ & 0.05 & $4.9(4.4-5.2)$ & $5.4(5.1-5.7)$ & $0.04^{\star}$ \\
\hline BLS-EMS & $5.8(5.1-6.2)$ & $5.1(4.2-5.8)$ & 0.16 & $5.4(4.2-6.0)$ & $5.5(5.0-5.9)$ & 0.49 \\
\hline ACLS-EMS & $6.1(5.7-6.5)$ & $5.6(4.7-6.1)$ & 0.06 & $6.0(5.1-6.5)$ & $5.8(5.4-6.3)$ & 0.69 \\
\hline \multicolumn{7}{|c|}{ Rate accuracy (\%) } \\
\hline BLS-ATS & $45.1(14.3-89.8)$ & $61.5(17.2-84.3)$ & 0.73 & $38.9(10.3-92.8)$ & $61.3(26.4-72.1)$ & 0.86 \\
\hline BLS-EMS & $64.9(14.3-79.3)$ & $57.7(39.4-85.1)$ & 0.82 & $70.0(7.4-87.5)$ & $57.8(50.5-70.9)$ & 0.60 \\
\hline ACLS-EMS & $0.0(0.0-71.7)$ & $14.3(0.1-68.2)$ & 0.42 & $0.2(0.0-76.7)$ & $30.5(0.0-64.2)$ & 0.76 \\
\hline \multicolumn{7}{|l|}{ Rate (cpm) } \\
\hline BLS-ATS & $110(100-125)$ & $106(100-123)$ & 0.69 & $112(97-124)$ & $105(100-125)$ & 0.95 \\
\hline BLS-EMS & $104(100-125)$ & $106(100-120)$ & 1.00 & $110(102-126)$ & $101(99-115)$ & 0.17 \\
\hline ACLS-EMS & $137(105-141)$ & $130(104-138)$ & 0.58 & $136(112-141)$ & $126(100-138)$ & 0.29 \\
\hline
\end{tabular}

Wilcoxon test, median (IQR).

FSP ON includes both FSP and FSP+feedback conditions, and FSP OFF includes both baseline and feedback conditions.

Feedback ON includes both feedback and FSP+feedback conditions, and feedback OFF includes both baseline and FSP conditions. * $p<0.05$ significant.

ACLS, advanced cardiovascular life support; ATS, athletic training students; BLS, basic life support; cpm, compression per min; EMS, emergency medical service; FSP, football shoulder pads.

2 min at the longest and practising removing the anterior portion or cutting the laces of FSP during ongoing chest compressions as medically trained personnel were able to perform high-quality CPR with the presence of FSP at least for the first $2 \mathrm{~min}$. Practising these skills in single rescuer situations is important. Not all public secondary schools have multiple athletic trainers as medical coverage during games and practices ${ }^{24}$ and treatment is situation dependent during cardiac arrest. In the first 2 min immediately after collapsing, we emphasise the importance of initiating chest compressions on the FSP without hesitating when there are complications with removing protective equipment. If an athletic trainer is able to seek the help of others, initiating chest compressions over the FSP for even the first $1 \mathrm{~min}$ or $30 \mathrm{~s}$ should be considered while others such as coaches, umpires and players prepare to help and all necessary tools are made available. For single rescuer situations during American football, we suggest the following protocol: (1) verify the scene safety and approach the collapsed athlete; (2) check the level of consciousness; (3) call for the help, activate EMS and ask to grab AED from sideline; (4) look for no breathing or no chest movement and check pulse; (5) initiate chest compression over the FSP until others get ready to help; (6) ask others to cut uniform and remove side strap of shoulder pads during ongoing chest compression; (7) remove hands from the chest and cut the anterior portion of shoulder pads with minimising chest fraction time; (8) resume chest compression within $10 \mathrm{~s}$; (9) apply AED during ongoing chest compression.

The numbers of each group were limited due to the small size of the studied groups. The study was limited to the measurement of recoil. The actual high-quality CPR defined by AHA was depth, rate and recoil. AED Plus and Real CPR Help were not able to measure recoil, so the actual high-quality CPR was not able to be measured from this study. Also, audio feedback was limited to 'push harder' when the depth was lower than $5 \mathrm{~cm}$ and no audio feedback was given when the depth went beyond $6 \mathrm{~cm}$. On the AED device, depth accuracy would automatically be calculated at $0 \%$ when participants compressed below $5.0 \mathrm{~cm}$ or above $6.0 \mathrm{~cm}$. One recent study reported the compression rate was $106.6 \pm 17.5 \mathrm{cpm}$ with the metronome sound at 100 times and $112.0 \pm 17.1 \mathrm{cpm}$ without the metronome sound.$^{27} \mathrm{~A}$ lower mean compression rate was reported of 103.1 cpm with audiovisual feedback and $108.0 \mathrm{cpm}$ without feedback, but the IQR was between 96 and $110 \mathrm{cpm}$ with audiovisual feedback, and 99 and $117 \mathrm{cpm}$ without feedback. ${ }^{16}$ A previous study indicated that with audio feedback the compression rate could get closer to the rhythm at which the sound was set, indicating the 
possibility of being slightly lower or higher than the setting rhythm. From our study, 106(100-129) cpm with audio feedback was found but the rate accuracy was 59.2 $(17.3-74.3) \%$ with audio feedback and $17.1(0-80.7) \%$ without feedback (figure 2). Again, rate accuracy would automatically be calculated at $0 \%$ when participants compressed at below $100 \mathrm{cpm}$ or above $120 \mathrm{cpm}$. Visual feedback clearly showed the targeted depth of $5-6 \mathrm{~cm}$. For depth feedback, Real CPR Help followed the 2010 AHA guidelines for audio feedback, but visual feedback was given at $5-6 \mathrm{~cm}$ according to the 2015 AHA guidelines. Real CPR Help gives audio feedback, such as 'push harder', 'good compressions' and 'continue CPR'. Visual feedback could be immediately seen in the performance of compression depth, whereas audio feedback on rate was given throughout the entire $2 \mathrm{~min}$. Only the metronome beep was set at $100 \mathrm{cpm}$ and no visual feedback was given for rate feedback. Our study did not run statistical correlational procedures to identify whether or not the number of BLS courses attended related to performance in the studied population. A future study may focus on the relationship between CPR performance and experience in BLS courses.

\section{CONCLUSION}

The certification level did not affect CPR performance. As long as a participant had taken a BLS course before, the performance levels were similar. No difference was observed between the results to suggest dissimilarity of CPR performance levels between first responders and EMS personnel. The use of a feedback device during chest compression-only CPR resulted in delivering a higher quality of CPR suggesting feedback devices must be introduced into athletic training settings. Regardless of the presence of a feedback device, the chest compression depth became shallower in CPR with FSP, but the depth remained within the 2015 AHA guidelines, and, in fact, the CPR quality did not change with the presence of FSP. The chest compression rate was not affected by the presence of both the feedback device and FSP. In case of a manpower shortage, athletic trainers should immediately initiate chest compressions over the FSP at least for the first 2 min until bystanders and AEDs are ready.

Acknowledgements The authors thank the Zoll Medical Cooperation for assistance in using the AED Pro and Real CPR Help.

Contributors ST, WR and SS contributed to the study design. ST carried out the all studies, participated in the sequence alignment and drafted the manuscript. HT helped to provide the intervention equipment. All authors performed the data analysis. WR, SS, HT and RS advised and helped to revise the manuscript. ST prepared the manuscript and all authors read and approved the final manuscript.

\section{Competing interests None declared.}

Provenance and peer review Not commissioned; externally peer reviewed.

Open Access This is an Open Access article distributed in accordance with the Creative Commons Attribution Non Commercial (CC BY-NC 4.0) license, which permits others to distribute, remix, adapt, build upon this work noncommercially, and license their derivative works on different terms, provided the original work is properly cited and the use is non-commercial. See: http:// creativecommons.org/licenses/by-nc/4.0/

Correction notice This paper has been amended since it was published Online First. Owing to a scripting error, some of the publisher names in the references were replaced with 'BMJ Publishing Group'. This only affected the full text version, not the PDF. We have since corrected these errors and the correct publishers have been inserted into the references.

(C) Article author(s) (or their employer(s) unless otherwise stated in the text of the article) 2017. All rights reserved. No commercial use is permitted unless otherwise expressly granted.

\section{REFERENCES}

1. Mozaffarian D, Benjamin EJ, Go AS, et al. Heart disease and stroke statistics-2015 update: a report from the American Heart Association. Circulation 2015;131:e29-322.

2. Kitamura T, Iwami T, Kawamura T, et al. Time-dependent effectiveness of chest compression-only and conventional cardiopulmonary resuscitation for out-of-hospital cardiac arrest of cardiac origin. Resuscitation 2011;82:3-9.

3. Kitamura T, Iwami T, Kawamura T, et al. Nationwide public-access defibrillation in Japan. N Engl J Med 2010;362:994-1004.

4. Casa DJ, Guskiewicz KM, Anderson SA, et al. National athletic trainers' association position statement: preventing sudden death in sports. J Athl Train 2012;47:96-118.

5. Neumar RW, Shuster M, Callaway CW, et al. American Heart Association guidelines Update for Cardiopulmonary Resuscitation and Emergency Cardiovascular Care, Part 1: executive Summary. Circulation 2015;2015:S315-S367.

6. Stiell IG, Brown SP, Christenson J, et al. What is the role of chest compression depth during out-of-hospital cardiac arrest resuscitation? Crit Care Med 2012;40:1192-8.

7. Yang Z, Li H, Yu T, et al. Quality of chest compressions during compression-only CPR: a comparative analysis following the 2005 and 2010 american Heart Association guidelines. Am J Emerg Med 2014;32:50-4.

8. Edelson DP, Abella BS, Kramer-Johansen J, et al. Effects of compression depth and pre-shock pauses predict defibrillation failure during cardiac arrest. Resuscitation 2006;71:137-45.

9. Gruenerbl A, Pirkl G, Monger E, et al, 2015. Smart-watch life saver: smart-watch interactive-feedback system for improving bystander CPR. ISWC '15 Proceedings of the 2015 ACM International Symposium on Wearable Computer. New York, NY:ACM 19-26.

10. Stiell IG, Brown SP, Nichol G, et al. What is the optimal chest compression depth during out-of-hospital cardiac arrest resuscitation of adult patients? Circulation 2014;130:1962-70.

11. Perkins GD, Lall R, Quinn T, et al. Mechanical versus manual chest compression for out-of-hospital cardiac arrest (PARAMEDIC): a pragmatic, cluster randomised controlled trial. Lancet 2015;385:947-55.

12. Hellevuo $H$, Sainio $M$, Nevalainen $R$, et al. Deeper chest compression - more complications for cardiac arrest patients? Resuscitation 2013;84:760-5.

13. Lee $\mathrm{SH}$, Kim DH, Kang TS, et al. The uniform chest compression depth of $50 \mathrm{~mm}$ or greater recommended by current guidelines is not appropriate for all adults. Am J Emerg Med 2015;33:1037-41.

14. Kramer-Johansen J, Myklebust H, Wik L, et al. Quality of out-ofhospital cardiopulmonary resuscitation with real time automated feedback: a prospective interventional study. Resuscitation 2006;71:283-92.

15. Abella BS, Edelson DP, Kim S, et al. CPR quality improvement during in-hospital cardiac arrest using a real-time audiovisual feedback system. Resuscitation 2007;73:54-61.

16. Hostler D, Everson-Stewart S, Rea TD, et al. Effect of real-time feedback during cardiopulmonary resuscitation outside hospital: prospective, cluster-randomised trial. BMJ 2011;342((d512)):1-10.

17. Miller MG, Thompson G, Rice A, et al. The effects of visual feedback on CPR skill retention in graduate student athletic trainer. JSMAHS 2015;1:1-6.

18. Truszewski Z, Szarpak L, Kurowski A, et al. Randomized trial of the chest compressions effectiveness comparing 3 feedback CPR devices and standard basic life support by nurses. Am J Emerg Med 2016;34:381-5.

19. Krasteva V, Jekova I, Didon JP. An audiovisual feedback device for compression depth, rate and complete chest recoil can improve the CPR performance of lay persons during self-training on a manikin. Physiol Meas 2011;32:687-99.

20. Skorning M, Beckers SK, Brokmann JCh, JCh B, et al. New visual feedback device improves performance of chest compressions by 
professionals in simulated cardiac arrest. Resuscitation 2010;81:53-8.

21. Del Rossi G, Bodkin D, Dhanani A, et al. Protective athletic equipment slows initiation of CPR in simulated cardiac arrest. Resuscitation 2011;82:908-12.

22. Larsen MP, Eisenberg MS, Cummins RO, et al. Predicting survival from out-of-hospital cardiac arrest: a graphic model. Ann Emerg Med 1993;22:1652-8.

23. Waninger KN, Goodbred A, Vanic K, et al. Adequate performance of cardiopulmonary resuscitation techniques during simulated cardiac arrest over and under protective equipment in football. Clin J Sport Med 2014;24:280-3.
24. Pryor RR, Casa DJ, Vandermark LW, et al. Athletic training services in public secondary schools: a benchmark study. J Athl Train 2015;50:156-62.

25. Heidenreich JW, Berg RA, Higdon TA, et al. Rescuer fatigue: standard versus continuous chest-compression cardiopulmonary resuscitation. Acad Emerg Med 2006;13:1020-6.

26. Shin J, Hwang SY, Lee HJ, et al. Comparison of CPR quality and rescuer fatigue between standard 30:2 CPR and chest compressiononly CPR: a randomized crossover manikin trial. Scand J Trauma Resusc Emerg Med 2014;22:59.

27. Zimmerman E, Cohen N, Maniaci V, et al. Use of a Metronome in Cardiopulmonary Resuscitation: a Simulation Study. Pediatrics 2015;136:905-11. 